\title{
Longitudinal equilibrium density distribution of arbitrary filled bunches in presence of a passive harmonic cavity and the short range wakefield
}

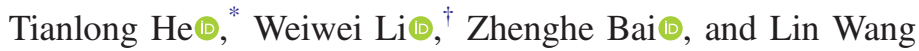 \\ National Synchrotron Radiation Laboratory, University of Science and Technology of China, \\ Hefei, Anhui, 230029, China
}

(Received 21 December 2020; accepted 16 March 2021; published 2 April 2021)

\begin{abstract}
We propose a novel scheme for the calculation of bunch profiles and synchronous phase shifts for arbitrary fill patterns in electron storage rings considering both the effects of the passive harmonic cavity and the short range wakefield. This scheme treats the bunch as a charge distribution rather than a point charge with a bunch form factor, and divides the voltage induced in the cavity into two groups: that induced by the previous passages and that by the present passage, where the latter is integrated with the short range wakefield from all the other vacuum components. An iteration loop for computing the bunch profiles, including a subloop using Newton iteration for calculating the synchronous phase deviations, is built and implemented into a MATLAB-based code. Using this code, we study the bunch lengthening and centroid transient effects for several possible fill patterns in the Hefei Advanced Light Facility storage ring under design. We find the method is very efficient, and the CPU time for the whole calculation on a regular personal computer is less than 30 seconds for each case even with more than 700 bunches. Using the case of a long gap fill as a benchmark example, both the results given by our code and the multiparticle tracking simulation with ELEGANT are in very good agreement.
\end{abstract}

DOI: 10.1103/PhysRevAccelBeams.24.044401

\section{INTRODUCTION}

In the ongoing fourth generation storage rings (also called diffraction-limited storage rings), the higher harmonic cavity (HHC) is an essential component to attain the machine performance, which can, by elongating the bunch, mitigate the intrabeam scattering, increase the Touschek lifetime, and reduce the beam-induced vacuum components heating [1]. In addition, the HHC, by flattening the potential at the synchronous phase to introduce synchrotron tune spread, can also provide Landau damping for the longitudinal coupled bunch instability induced by the higher order modes of the cavities [2,3]. In comparison to the active HHC driven by the external rf power, generally, the passive HHC, excited by the beam, is preferred by many machines [4-10], and thus focused on in this paper.

The bunch lengthening is significantly affected by the HHC settings and the beam-fill patterns. For the case of uniform fill pattern, the bunch length can be calculated analytically, as the total voltage including the loading

\footnotetext{
*htlong@ustc.edu.cn

†iwe@ustc.edu.cn
}

Published by the American Physical Society under the terms of the Creative Commons Attribution 4.0 International license. Further distribution of this work must maintain attribution to the author(s) and the published article's title, journal citation, and DOI. voltage of the $\mathrm{HHC}$ can be given by a simple analytical formula [11,12]. An optimum lengthening condition [11] is thus proposed and can be met by zero the first and second derivatives of the total voltage at the synchronous phase, where the bunch profile is flattop and the bunch length is increased to at least a factor of 4 . However, in reality, the nonuniform fill pattern is more common, since a long gap or some short gaps are usually introduced to suppress the ion instability and transverse coupled-bunch instabilities [13], and meet the requirements of the timing users [14] or the injection [15]. The gaps will generate the transient beam loading effect so that the stable voltages seen by the bunches are different from each other, which may cause much less bunch lengthening and also lead to the variation of the bunch lengths along the bunch trains. We can adopt a scheme to reduce the effects of the gaps with higher charges adjacent to the gaps (called "guard bunches") compensating for the charge missing in gaps [16,17].

Two types of approaches can be used to study the bunch lengthening for the case of nonuniform fill pattern: semianalytical calculation and multiparticle tracking simulation. The former can be traced back to [18], where the bunch was treated as a point charge, and the induced voltage seen by every bunch was determined by the single particle tracking, so the effect of the bunch profile was ignored. It was followed and later modified with considering the bunch profile by introducing the bunch form factor which is either scalar or complex $[14,19]$. The effects of the scalar and the 
complex bunch form factors were discussed in detail in [14]. In addition to the single particle tracking, a novel semianalytical method based on an iterative matrix formulation was also described in [14], to compute the equilibrium solution numerically. Recently, Warnock and Venturini [17] presented another novel robust and efficient semianalytical method which used a Newton iteration for a solution of a system of coupled Haïssinski equations which determine the bunch positions and profiles in the equilibrium state. Several multiparticle tracking codes, e.g., MBTRACK [20], ELEGANT [21], and SPACE [22], can be, of course, used to study the case of nonuniform fill pattern, for not only the steady state case but also the unsteady state case. Compared with the multiparticle-based methods, the semianalytical methods are much faster, and thus preferred when studying the steady state case.

In this paper, we propose an alternative novel semianalytical scheme to calculate the longitudinal equilibrium density distribution for arbitrary fill patterns and HHC settings. This scheme also uses the matrix formulation to form the induced voltage as done in [14], but treats the bunch as a charge density distribution rather than a macroparticle with a bunch form factor. In addition to the HHC, the bunch lengthening induced by the short range wakefield from the other vacuum chamber elements can also be considered. Our scheme is also to solve the Haïssinski equations numerically as done by Warnock and Venturini [17], but the algorithm is quite different. The parameters of the Hefei Advanced Light Facility (HALF) storage ring [23], which is designed at $2.2 \mathrm{GeV}$ with natural emittance lower than $100 \mathrm{pm}$, will be used to test the method.

The paper is organized as follows. Section II describes the Haïssinski equation in the presence of the double $\mathrm{rf}$ systems and the short range wakefield, which will be used for the calculation of the longitudinal density distribution. Section III introduces the induced voltage formed by a matrix formulation for arbitrary fill patterns. Section IV and Appendix B state a Newton iteration method for the calculation of the synchronous phase deviation. Section V shows the phasor plot used in this paper for the passive HHC, and how to obtain the voltage ratio and synchronous phase from the voltage phasor. Section VI describes the iteration loop and the detailed procedures. In Sec. VII, the near optimum lengthening condition is discussed. In Sec. VIII, the related parameters of HALF are given, the bunch profiles and centroid positions of several different fill patterns are discussed, and the comparison to the ELEGANT tracking results is also shown. Finally, conclusions and discussions are given in Sec. IX. As an extension, the phasor plot for the main cavity is presented in Appendix A, and the corresponding Newton iteration method for the synchronous phase deviation is described in Appendix B.

\section{POTENTIAL OF DOUBLE RF SYSTEMS AND THE SHORT RANGE WAKEFIELD}

For double rf systems, the total cavity voltage can be given by [12]

$V_{T}=V_{r f} \sin \left(\omega_{r f} \tau+\varphi_{s}\right)+k V_{r f} \sin \left(n \omega_{r f} \tau+\varphi_{h}\right)$,

where $V_{r f}, \omega_{r f}$ and $\varphi_{s}$ are the voltage amplitude, angular fundamental frequency and synchronous phase of the main cavity, respectively, $k$ is the voltage ratio of the harmonic cavity relative to the main cavity, and $n$ and $\varphi_{h}$ are the harmonic order and synchronous phase of the harmonic cavity, respectively.

Let $\rho(\tau)$ represent the longitudinal normalized density distribution of a bunch, then the equations of motion of a single particle in this bunch can be given by

$$
\begin{gathered}
\frac{d \tau}{d t}=\alpha_{c} \delta \\
\frac{d \delta}{d t}=\frac{e V_{T}-U_{0}}{E_{0} T_{0}}+\frac{q e}{E_{0} T_{0}} \int_{-\infty}^{\infty} \rho\left(\tau^{\prime}\right) W_{L}\left(\tau-\tau^{\prime}\right) d \tau^{\prime}
\end{gathered}
$$

where $(\tau, \delta)$ are the particle coordinates (respectively represent the time deviation and relative momentum deviation with respect to the synchronous particle), $\alpha_{c}$ the momentum compaction, $U_{0}$ the radiation loss per turn, $e$ the electron charge, $E_{0}$ the nominal energy, $T_{0}$ the revolution time, $q$ the total charge of this bunch, and $W_{L}$ the longitudinal short range wakefield function for all the vacuum chamber elements, including the rf cavities.

The corresponding Hamiltonian is then

$$
H=-\frac{1}{2} \delta^{2}+\Phi(\tau)
$$

where $\Phi(\tau)$ is the total longitudinal potential contributed by the double rf systems and the short range wakefield, and is given by

$$
\begin{aligned}
\Phi(\tau)= & \frac{e V_{r f}}{2 \pi h \alpha_{c} E_{0}}\left\{\cos \left(\varphi_{s}\right)-\cos \left(\omega_{r f} \tau+\varphi_{s}\right)\right. \\
& \left.+\frac{k}{n}\left[\cos \left(\varphi_{h}\right)-\cos \left(n \omega_{r f} \tau+\varphi_{h}\right)\right]\right\}-\frac{U_{0} \omega_{r f} \tau}{e V_{r f}} \\
& +\frac{q}{E_{0} T_{0} \alpha_{c}} \int_{0}^{\tau} d \tau^{\prime} \int_{-\infty}^{\infty} \rho\left(\tau^{\prime \prime}\right) W_{L}\left(\tau^{\prime}-\tau^{\prime \prime}\right) d \tau^{\prime \prime},
\end{aligned}
$$

where the last term on the right-hand side corresponds to the short range wakefield. Then the longitudinal equilibrium density distribution of this bunch has the form

$$
\rho(\tau)=\rho_{0} \exp \left[\frac{\Phi(\tau)}{\sigma_{\delta}^{2}}\right]
$$


where $\sigma_{\delta}$ is the relative energy spread and $\rho_{0}$ is a normalization constant which is given by

$$
\rho_{0}=\frac{1}{\int_{-\infty}^{\infty} \exp \left[\frac{\Phi(\tau)}{\sigma_{\delta}^{2}}\right] d \tau} .
$$

Different from the Eq. (11) in [14], here the potential of the short range wakefield is included in Eq. (5), thus, the longitudinal density distribution needs to be solved iteratively by combining the Eq. (5)-(7), which is equivalent to solving the Haïssinski equation.

For the passive harmonic cavity and arbitrary fill patterns, as the equilibrium condition is assumed, every bunch will see its own steady voltage phasor induced by all circulating bunches. If the steady voltage phasor of every bunch can be obtained, then the ratio $k$, phase $\varphi_{h}$ and $\varphi_{s}$ can be calculated, as well as the density distribution. In the following sections, the steady voltage phasor will be introduced first, then the algorithm for the ratio $k$, phase $\varphi_{h}$ and $\varphi_{s}$.

\section{BEAM INDUCED VOLTAGE}

\section{A. Single bunch and one passage}

Considering a charged particle $q$ passing an rf cavity with resonant frequency $\omega_{r}$, loaded shunt impedance $R_{L}$ and loaded quality factor $Q_{L}$, the voltage in the cavity immediately after the particle has passed is given by $V_{q}=q \omega_{r} R_{L} / Q_{L}$, then $V_{q}$ will oscillate with the cavity resonant frequency and decay exponentially with time. If the complex voltage phasor concept [24] is used here, then the induced voltage phasor evolved with time $t$ can be expressed by

$$
\tilde{V}_{q}(t)=\frac{q \omega_{r} R_{L}}{Q_{L}} \exp \left[\left(j-\frac{1}{2 Q_{L}}\right) \omega_{r} t\right],
$$

where the real part of this voltage phasor represents the real voltage seen by the trailing point charges, and its positive value means energy loss.

When considering a bunch, with normalized charge density distribution $\rho_{i}(\tau)$, in the bucket $i$, the induced complex voltage seen by the synchronous particle of this bunch becomes

$\tilde{V}_{i, 0}=\frac{q_{i} \omega_{r} R_{L}}{Q_{L}} \int_{-\infty}^{\infty} \rho_{i}(\tau) \exp \left[\left(-j+\frac{1}{2 Q_{L}}\right) \omega_{r} \tau\right] d \tau$,

where $q_{i}$ is the bunch charge. The bunch length is usually much shorter than the wavelength of the cavity and $Q_{L}$ is usually much greater than 1 , so $\frac{\omega_{r} \tau}{2 Q_{L}} \ll 1$, and Eq. (9) can be approximated as

$$
\tilde{V}_{i, 0} \approx \frac{q_{i} \omega_{r} R_{L}}{Q_{L}} \int_{-\infty}^{\infty} \rho_{i}(\tau) \exp \left(-j \omega_{r} \tau\right) d \tau,
$$

where $\int_{-\infty}^{\infty} \rho_{i}(\tau) \exp \left(-j \omega_{r} \tau\right) d \tau$ is defined as the bunch form factor. Similar to Eq. (8), the bunch voltage phasor evolved with time is then given by

$$
\tilde{V}_{i}(t)=\tilde{V}_{i, 0} \exp \left[\left(j-\frac{1}{2 Q_{L}}\right) \omega_{r} t\right] .
$$

\section{B. Stable voltage induced by single bunch}

Now let us calculate the stable voltage induced by multipassages of one bunch through the rf cavity. For the first passage, the induced voltage phasor is $\tilde{V}_{i, 0}$ as mentioned above. For the second passage, the total voltage phasor becomes $\tilde{V}_{i, 0}+\tilde{V}_{i, 0} \exp \left[\left(j-\frac{1}{2 Q_{L}}\right) \omega_{r} T_{0}\right]$, where $T_{0}$ is the revolution time. After enough passages, the total voltage phasor becomes convergent and can be given by

$$
\tilde{V}_{i, \infty}=\frac{\tilde{V}_{i, 0}}{\left.1-\exp \left[\left(j-\frac{1}{2 Q_{L}}\right) \omega_{r} T_{0}\right)\right]} .
$$

For convenience, we define a same reference phase $\varphi_{s 0}$ of the main cavity for all the bunches and the synchronous phase for the bunch located in the bucket $i$ is given by $\varphi_{i}=\varphi_{s 0}+\Delta \varphi_{i}$, where $\Delta \varphi_{i}$ is the phase deviation with respect to the reference phase. Then the voltage phasor induced by the bunch in the bucket $i$ and seen by the synchronous particle in the bucket $k$ is

$$
\tilde{V}_{i \rightarrow k}=\tilde{V}_{i, \infty} \exp \left[\Delta_{i, k}+j n\left(\Delta \varphi_{k}-\Delta \varphi_{i}\right)\right],
$$

where $\Delta_{i, k}$ is given by

$$
\Delta_{i, k}= \begin{cases}\left(j-\frac{1}{2 Q_{L}}\right)(k-i) \omega_{r} T_{b} & \text { for } k>i \\ \left(j-\frac{1}{2 Q_{L}}\right)(k-i+h) \omega_{r} T_{b} & \text { for } k \leq i,\end{cases}
$$

where $T_{b}$ is the duration between two adjacent buckets and equal to $T_{0} / h, h$ is the harmonic number, $i$ and $k$ are integers in the range of $1 \sim h$. Note that we intend to treat the influence of the newly induced field during the present passage of the bunch $i$ through the cavity on itself as a kind of short range wakefield effect, so the evolution time for $\Delta_{i, i}$ is $T_{0}$ rather than 0 .

\section{Stable voltage induced by multibunches}

For convenience of expression, we treat the empty bucket as that filled with a zero charge bunch. The voltage phasors seen by the synchronous particles of all the bunches can form a matrix: 


$$
\left|\begin{array}{ccccc}
\tilde{V}_{1, \infty} e^{\Delta_{1,1}+j n\left(\Delta \varphi_{1}-\Delta \varphi_{1}\right)} & \cdots & \tilde{V}_{1, \infty} e^{\Delta_{1, k}+j n\left(\Delta \varphi_{k}-\Delta \varphi_{1}\right)} & \cdots & \tilde{V}_{1, \infty} e^{\Delta_{1, h}+j n\left(\Delta \varphi_{h}-\Delta \varphi_{1}\right)} \\
\vdots & \vdots & \vdots & \vdots & \vdots \\
\tilde{V}_{i, \infty} e^{\Delta_{i, 1}+j n\left(\Delta \varphi_{1}-\Delta \varphi_{i}\right)} & \cdots & \tilde{V}_{i, \infty} e^{\Delta_{i, k}+j n\left(\Delta \varphi_{k}-\Delta \varphi_{i}\right)} & \cdots & \tilde{V}_{i, \infty} e^{\Delta_{i, h}+j n\left(\Delta \varphi_{h}-\Delta \varphi_{i}\right)} \\
\vdots & \vdots & \vdots & \vdots & \vdots \\
\tilde{V}_{h, \infty} e^{\Delta_{h, 1}+j n\left(\Delta \varphi_{1}-\Delta \varphi_{h}\right)} & \cdots & \tilde{V}_{h, \infty} e^{\Delta_{h, k}+j n\left(\Delta \varphi_{k}-\Delta \varphi_{h}\right)} & \cdots & \tilde{V}_{h, \infty} e^{\Delta_{h, h}+j n\left(\Delta \varphi_{h}-\Delta \varphi_{h}\right)}
\end{array}\right|,
$$

where the element with row $i$ and column $k$ represents the voltage phasor induced by the bunch in the bucket $i$ and seen by the synchronous particle in the bucket $k$. Then the total voltage phasor seen by the synchronous particle in the bucket $k$ can be given by the sum of the elements of column $k$,

$$
\tilde{V}_{k}=\sum_{i=1}^{h} \tilde{V}_{i, \infty} \exp \left[\Delta_{i, k}+j n\left(\Delta \varphi_{k}-\Delta \varphi_{i}\right)\right] .
$$

Note that the voltage induced by the present passage is not included in Eq. (16), which has already been integrated into the short range wakefield group in Eqs. (3) and (5). The discussion in this subsection can also be adapted to consider the beam loading voltage of the main cavity and one only needs to take $n=1$ correspondingly.

\section{SYNCHRONOUS PHASE DEVIATION}

The synchronous phase deviation $\Delta \varphi_{i}$ is required for computing the voltage phasor in Eq. (16). Based on the fact that the energy gain of the synchronous particle per turn is zero, another equation can be given by

$$
\begin{aligned}
& V_{r f} \sin \left(\varphi_{s 0}+\Delta \varphi_{i}\right) \\
& -\operatorname{Re}\left\{\sum_{k=1}^{h} \tilde{V}_{k, \infty} \exp \left[\Delta_{k, i}+j n\left(\Delta \varphi_{i}-\Delta \varphi_{k}\right)\right]\right\} \\
& +q_{i} \int_{-\infty}^{\infty} \rho_{i}\left(\tau^{\prime}\right) W_{L}\left(0-\tau^{\prime}\right) d \tau^{\prime}-\frac{U_{0}}{e}=0,
\end{aligned}
$$

where the first term on the left-hand side represents the kick voltage in the main cavity, the second term represents the voltage induced by all the previous bunch passages through the passive harmonic cavity, and the third term represents the voltage generated by the short range wakefield. Besides, the present bunch passage though the harmonic cavity will also contribute to the third term.

To compute the synchronous phase deviation with a set of guess values $\rho_{i}(\tau)$, the system of the nonlinear Eqs. (17) for $i=1,2, \ldots h$ can be dealt well with by the Newton iteration method [25]. The details are given in Appendix B.

\section{V. $k$ AND $\varphi_{h}$ OF HARMONIC CAVITY}

Under the steady state condition, the stable voltage phasor $\tilde{V}_{i}^{h c}$, as shown in Fig. 1, is determined by Eq. (16). Then the real voltage seen by the bunch particles can be given by

$$
\left|\tilde{V}_{i}^{h c}\right| \cos \left(\psi_{h}-n \varphi\right)=k V_{r f} \sin \left(n \varphi+\frac{\pi}{2}-\psi_{h}\right) .
$$

Thus we have

$$
\begin{aligned}
k & =\frac{\left|\tilde{V}_{i}^{h c}\right|}{V_{r f}} \\
\psi_{h} & =\arctan \left(\frac{\operatorname{Re}\left[\tilde{V}_{i}^{h c}\right]}{\operatorname{Im}\left[\tilde{V}_{i}^{h c}\right]}\right) \pm \frac{\pi}{2},
\end{aligned}
$$

where $+(-)$ is taken when $\operatorname{Im}\left[\tilde{V}_{i}^{h c}\right]>0(<0)$, the synchronous phase $\varphi_{h}$ is easily obtained by $\varphi_{h}=\frac{\pi}{2}-\psi_{h}$ according to Eqs. (1) and (18).

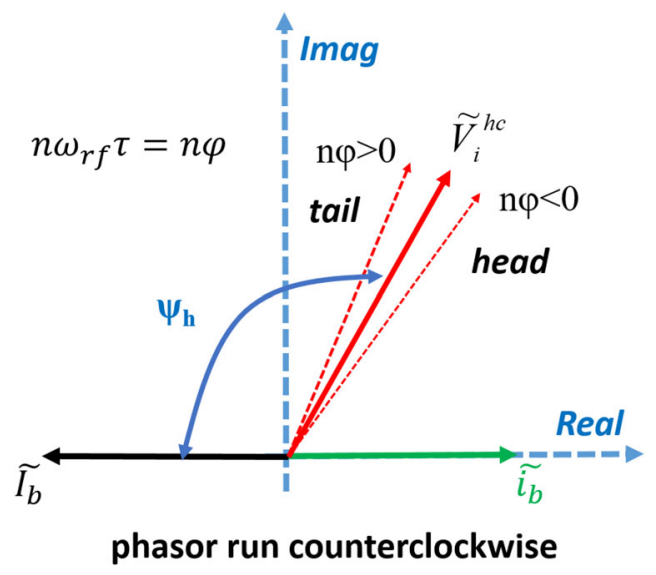

FIG. 1. Phasor diagram for harmonic cavity. The image current phasor $\tilde{i}_{b}$ is along the positive direction of the real axis, all phasors run counterclockwise, $\psi_{h}$ is the detuning phase of harmonic cavity, $\tilde{V}_{i}{ }^{h c}$ is the stable voltage phasor seen by the synchronous particle, and $n \varphi$ is the phase deviation of nonsynchronous particle from the synchronous particle, whose positive (negative) value corresponds to the bunch tail (head). 


\section{ITERATIVE LOOP}

An iterative loop can be established to calculate effectively the bunch profiles and synchronous phase deviations using the related equations in the above sections, as shown in Fig. 2, where the initial density distribution $\rho_{i}^{0}$ is set to a Gaussian distribution with the natural bunch length at the zero current limit, and the deviation of the synchronous phase $\Delta \varphi_{i}^{0}$ is set to zero. To make an economical calculation, the reference phase $\varphi_{s 0}$ is chosen by

$$
\begin{gathered}
V_{r f} \sin \left(\varphi_{s 0}\right)-2 F I_{0} R_{L} \cos \left(\psi_{h}\right) \cos \left(\psi_{h}\right)=\frac{U_{0}}{e}, \\
\tan \left(\psi_{h}\right)=-2 Q_{L} \frac{\Delta \omega_{r}}{n \omega_{r f}},
\end{gathered}
$$

where $\Delta \omega_{r}=\omega_{r}-n \omega_{r f}$ is the detuning angular frequency of harmonic cavity, $I_{0}$ is the average beam current, and $F \approx 1$ for short bunches. $\varphi_{s 0}$ is also an approximate solution to the case of uniform fill pattern.

Updated synchronous phase deviation $\Delta \varphi_{i}^{1}$ can be obtained using the Newton iteration method in Eq. (17), and the corresponding voltage phasor $\tilde{V}_{i}$ using Eq. (16). Once $\Delta \varphi_{i}^{1}$ and $\tilde{V}_{i}$ are obtained, Eq. (19) is solved to get $k_{i}$ and $\psi_{h, i}$, and the synchronous phase $\varphi_{i}^{1}$ is equal to $\varphi_{s 0}+\Delta \varphi_{i}^{1}$. Then the current density distribution $\rho_{i}^{1}$ is updated by Eqs. (5)-(7).

If the maximum relative deviation between the current and the previous distributions is less than the allowable set error $\left(10^{-5}\right.$ for our cases), the loop will be terminated and the results will be outputted, otherwise, the loop will continue. In order to speed up the convergence and avoid the occurrence of cyclic solutions, before starting the next round of calculation, the current density distributions are updated and weighted by a random coefficient with the previous and the current distributions.

The iteration loop has been implemented into a MATLABbased code, which was found to be very efficient for hundreds of arbitrary filled bunches, e.g., for HALF cases with a maximum bunch number of 800 , the elapsed time is within 30 seconds. In Sec. VIII, the cases of HALF storage ring with several possible fill patterns will be shown to demonstrate the capability of this code.

\section{NEAR OPTIMUM CONDITION}

For the case of uniform fill, the induced voltage ratio in the harmonic cavity is given by [12]

$$
k=\frac{-2 F I_{0} R_{L} \cos \left(\psi_{h}\right)}{V_{r f}} .
$$

The optimum lengthening condition is proposed by making both the first and second order derivatives of the total voltage be zero, which is also called flat potential conditions. This is achieved when the harmonic cavity voltage ratio and phase are [11]

$$
\begin{aligned}
k_{f p} & =\sqrt{\frac{1}{n^{2}}-\frac{1}{n^{2}-1}\left(\frac{U_{0}}{e V_{r f}}\right)^{2}}, \\
\tan \varphi_{h, f p} & =-\frac{n U_{0}}{e V_{r f}} \frac{1}{\sqrt{\left(n^{2}-1\right)^{2}-\left(\frac{n^{2} U_{0}}{e V_{r f}}\right)^{2}}} .
\end{aligned}
$$

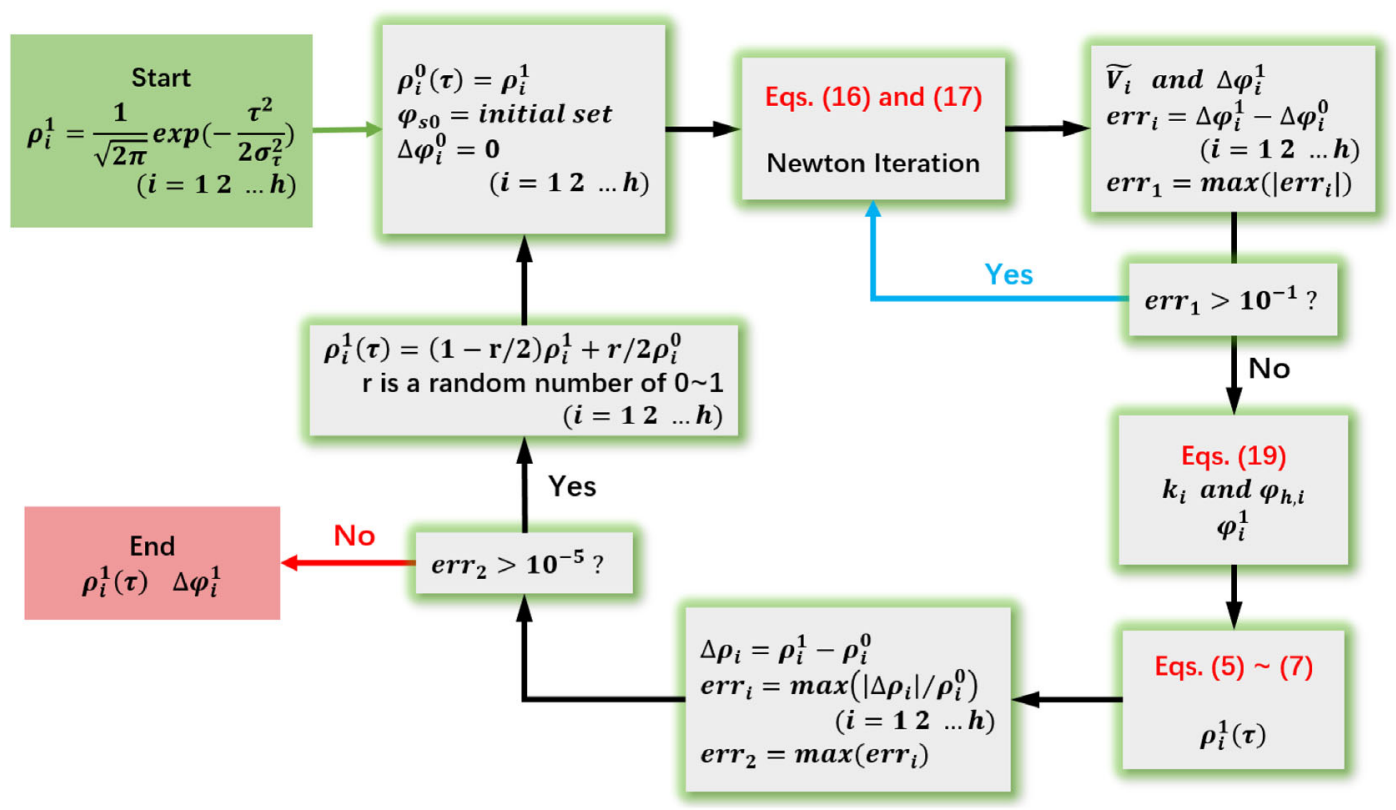

FIG. 2. The iterative loop diagram, including a big loop for computing the longitudinal density distribution and a subloop of Newton iteration for calculating the synchronous phase deviation. 
However, for the target current and the determined shunt impedance, the flat potential conditions cannot always be met due to the mismatch of the shunt impedance, especially for the superconducting HHC [26]. That is to say, either the voltage or the phase condition can be reached by adjust the detuning frequency of the passive harmonic cavity. So we will use Eqs. (21)-(23) to determine the detuning frequency (which we call the near optimum lengthening condition). This condition may lead to a significant deformation of the bunch shape which is far away from the flattop profile. Nevertheless, we can still use it to choose the detuning frequency since it can still reach relative fine results of bunch lengthening. The detuning frequency will be also used in the below sections for the case of nonuniform fill.

\section{NUMERICAL RESULTS FOR THE PARAMETERS OF HALF}

A tentative set of parameters of HALF storage ring without insertion devices is summarized in Table I, where the harmonic cavity is assumed to have the same quality factor $Q$ as that of ALS [18], but has a lower $R / Q$ of $60 \Omega$ (in circuit definition). Using these parameters, several fill patterns, including uniform, a long gap and uniformly distributed gaps, will be calculated and discussed. The continuous density distribution of each bunch is calculated at discrete sampling points uniformly distributed in the range of $\tau \in[-150,150]$ ps. We take 300 points here and even if we increase the number of the points further the changes of calculation results are very slight by graphical comparisons.

The main cavity is assumed to be ideal, as others did $[14,17]$, since we only focus on the beam loading effect of harmonic cavity in this paper. In reality, the beam loading and the generator voltages of the main cavity will affect each other through a complex rf feedback. Assuming a given generator voltage, our method can be extended to include the beam loading effect of the main cavity, and the

TABLE I. Main parameters of HALF.

\begin{tabular}{lcc}
\hline \hline Parameter & Symbol & Value \\
\hline Ring circumference & $\mathrm{C}$ & $480 \mathrm{~m}$ \\
Beam energy & $E_{0}$ & $2.2 \mathrm{GeV}$ \\
Nominal beam current & $I_{0}$ & $350 \mathrm{~mA}$ \\
Longitudinal damping time & $\tau_{z}$ & $20 \mathrm{~ms}$ \\
Momentum compaction & $\alpha_{c}$ & $6.3 \times 10^{-5}$ \\
Natural energy spread & $\sigma_{\delta}$ & $6.6 \times 10^{-4}$ \\
Harmonic number & $\mathrm{h}$ & 800 \\
Energy loss per turn & $U_{0}$ & $218 \mathrm{keV}$ \\
Voltage of MC & $V_{r f}$ & $0.746 \mathrm{MV}$ \\
Natural rms bunch length & $\sigma_{t 0}$ & $6.67 \mathrm{ps}$ \\
Harmonic number of HHC & $\mathrm{n}$ & 3 \\
Detuning frequency of HHC & $\Delta f$ & $128.3 \mathrm{kHz}$ \\
Shunt impedance of HHC & $R_{s}$ & $1.26 \mathrm{M} \Omega$ \\
Quality factor of HHC & $\mathrm{Q}$ & 21000 \\
\hline \hline
\end{tabular}

details of the algorithm can be found in Appendixes A and $\mathrm{B}$.

In subsections below, only the wakefield from the HHC is considered except for Secs. VIII E and VIII F, where the short range wakefield from all other vacuum components is represented by a broadband resonator model.

\section{A. Uniform fill with 800 bunches}

We first study the case of uniform and complete fill pattern at the nominal current of $350 \mathrm{~mA}$. Figure 3 shows the resulting charge density profiles of 800 identical bunches. The detuning frequency determined by the near optimum condition is $128.3 \mathrm{kHz}$. By decreasing the detuning frequency, it is expected that the bunch can be lengthened to more than a factor of 5. As seen in Fig. 3, with decreasing the detuning frequency from 133.3 to $120.3 \mathrm{kHz}$, the rms bunch length is increased from 22.8 to $34.6 \mathrm{ps}$, but the charge density distribution becomes more and more asymmetric. The flattop potential conditions require the shunt impedance be $2.93 \mathrm{M} \Omega$, while the shunt impedance of $1.26 \mathrm{M} \Omega$ which we used is far away from this value. So we achieve neither a flattop nor two maxima in the density like that in [17].

\section{B. Train with a single long gap}

Next, we consider a train with a single long gap (a number of successive empty buckets), which can be used for mitigating the ion instability or meeting the requirements of some synchrotron light users. An example of a bunch train with 80 empty buckets is studied using the parameters listed in Table I. Several representative bunch forms near the front, middle, and end of the train are shown in Fig. 4.

We also consider the impact of $R / Q$ on the bunch lengthening since the transient effect scales as its value [18]. Figure 5 shows the centroid positions and rms lengths for $R / Q$ of 60,100 and $140 \Omega$, respectively, where the $Q$

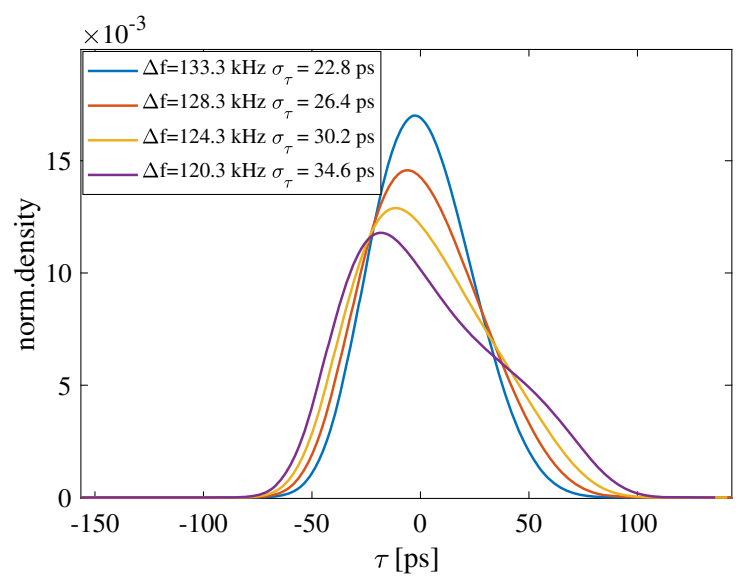

FIG. 3. Charge density profile for uniform fill with 800 identical bunches at $350 \mathrm{~mA}$. 


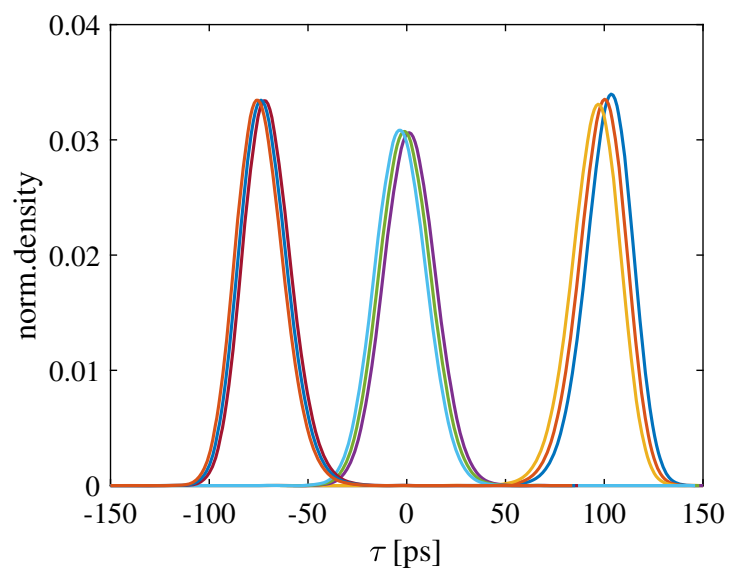

FIG. 4. Results for the long gap fill with 720 identical bunches. The right three curves correspond to bunch 1, 11 and 21; the middle three to 350,360 and 370; the left three to 700, 710 and 720 .

values are all $2.1 \times 10^{4}$ and the detuning frequencies are determined by the near optimum lengthening condition. Comparing with the uniform fill pattern, there is much less bunch lengthening and a larger centroid shift varying

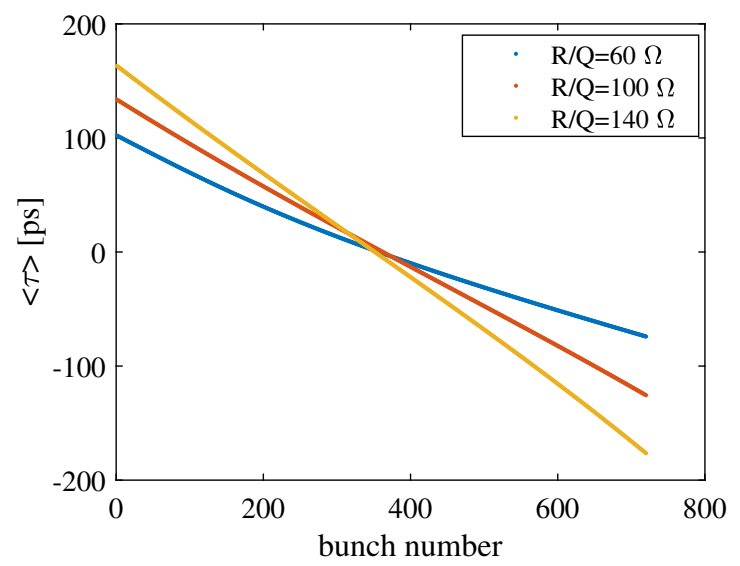

(a)

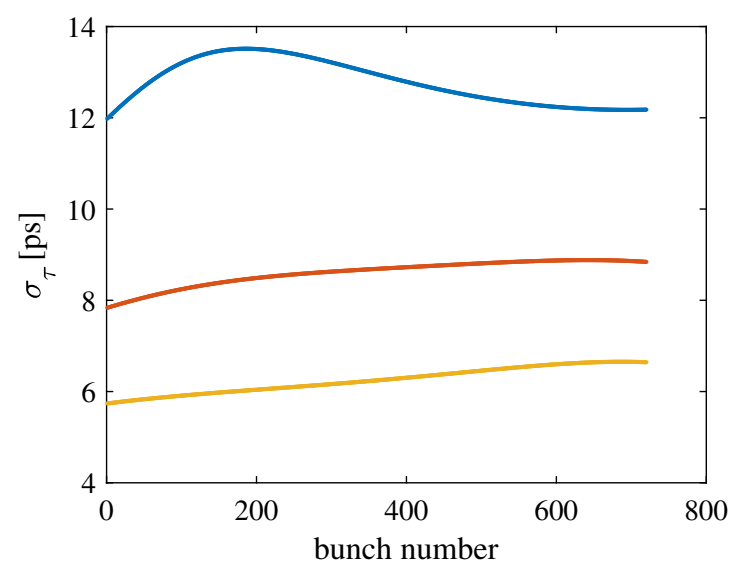

(b)

FIG. 5. Centroid position (a) and rms bunch length (b) vs bunch number. linearly along the train for each case. But it also indicates that a small $R / Q$ would be helpful for mitigating the transient effect. As can be seen in Fig. 5, the bunches can still be lengthened to a factor of about 2 with $R / Q=60 \Omega$, while the bunches are even slightly shortened with $R / Q=140 \Omega$.

\section{Compensation for the long gap}

For improving the bunch lengthening performance, the scheme based on the guard bunches can be used to compensate for the long gap in the fill pattern [14,17]. We consider the case of 25 bunches on both sides of the gap with 3 times nominal charge while maintaining the average current of $350 \mathrm{~mA}$. Figure 6 shows the results for several representative bunch profiles. Figure 7 shows the centroid position and the rms length vs bunch number.

We can see that the transient effect is reduced obviously and the overall bunch lengthening performance is effectively improved. The middle three bunches are near flattop and lengthened to a factor of about 5. The head and tail bunches are near Gaussian and lengthened to a factor of about 2 . However, the highly intense guard bunches could suffer from the microwave instability, or a reduced lifetime, or some other undesirable collective effects.

\section{Uniformly distributed gaps}

The fill pattern with a single long gap could suffer from heavy transient beam loading effect and lead to an undesirable bunch lengthening performance, even if the guard bunches are used to compensate the gap. Thus we consider the fill pattern with uniformly distributed gaps for HALF. This has a chance of resembling more closely the complete fill. Keeping the average current of $350 \mathrm{~mA}$, the 800 buckets are divided into 20 periods, each with 36 successive filled bunches and four empty buckets.

The resulting bunch parameters also turn out to be periodic in bucket number, as shown in Fig. 8. Figure 9

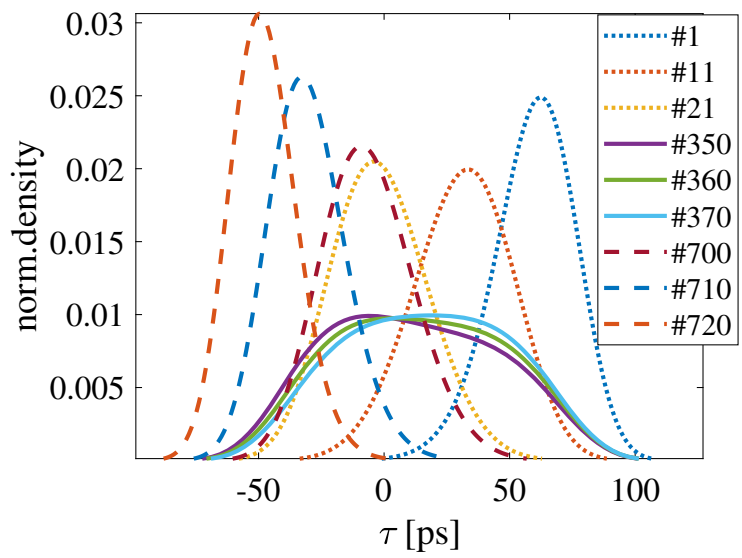

FIG. 6. Charge density profile. Nine bunches are chosen to be shown from the head, middle and tail parts of the bunch train. 


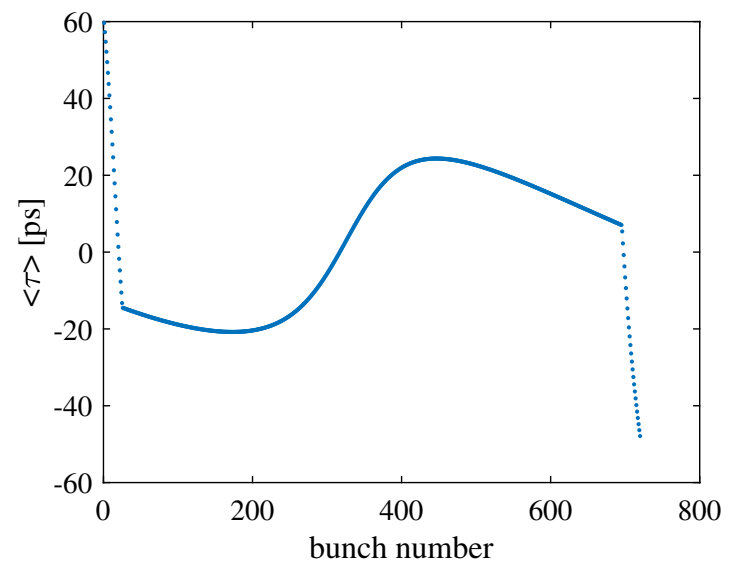

(a)

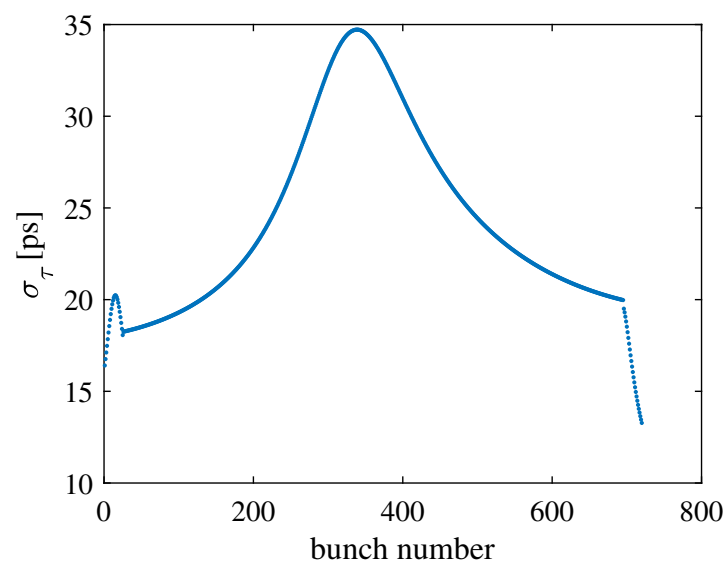

(b)

FIG. 7. Centroid position (a) and rms bunch length (b) vs bunch number, with guard bunches.

displays the normalized density profiles of several representative bunches in the first bunch train. The centroid displacement is much smaller in comparison to that of the long gap case, and its magnitude is similar to that of the complete fill. The ratio of the average rms length over all the bunches to the natural bunch length is about 4, which is near the case of uniform fill.

\section{E. Uniformly distributed gaps with a broadband resonator impedance}

As is known, besides the harmonic cavity, the short range wakefield from other vacuum components can also contribute to bunch lengthening, which was neglected in the above discussions but can be naturally included in our semianalytical scheme. To illustrate such capability of the proposed approach, we study the case presented in Sec. VIIID again but with the short range wakefield considered. For simplicity, the short range wakefield is represented by a single broadband resonator (BBR) model with resonant frequency of $30 \mathrm{GHz}$, quality factor of 1 and shunt impedance of $3 \mathrm{k} \Omega$. The results are shown in Figs. 10 and 11 .

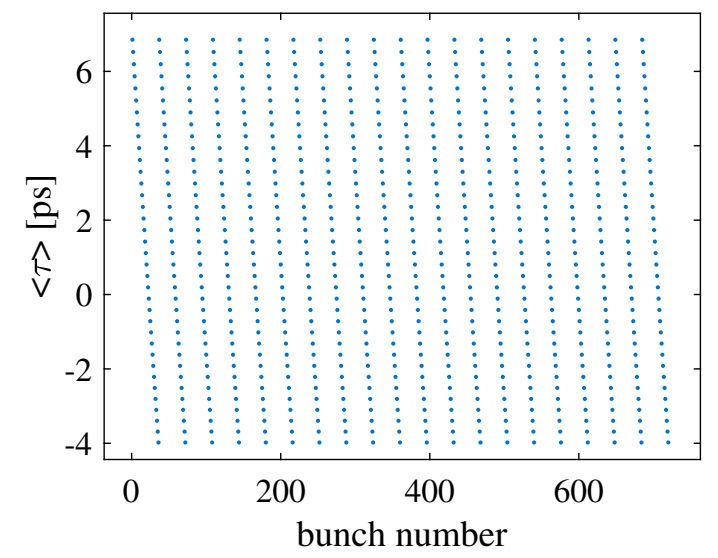

(a)

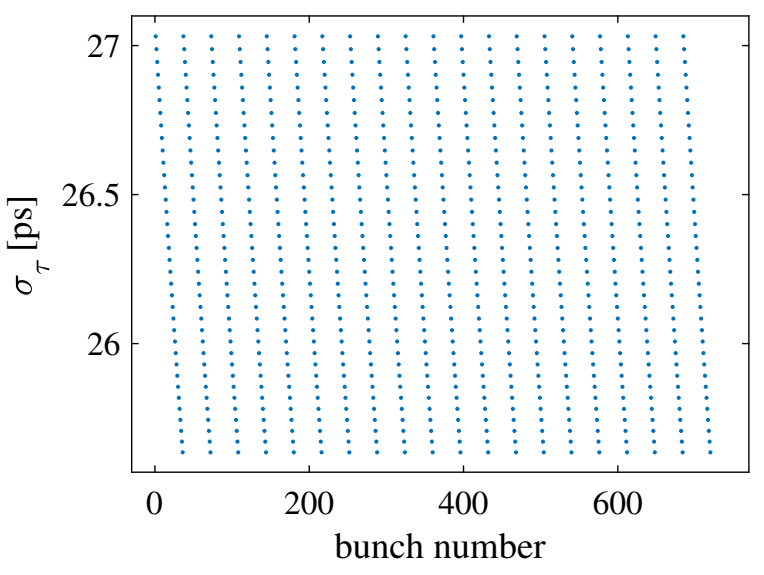

(b)

FIG. 8. Centroid position (a) and rms bunch length (b) vs bunch number, with uniform distributed gaps.

Comparing to those in Figs. 8 and 9, there is a smaller centroid displacement and variation of bunch length along each subtrain, and the average rms length over all the bunches reaches 30.4 ps.

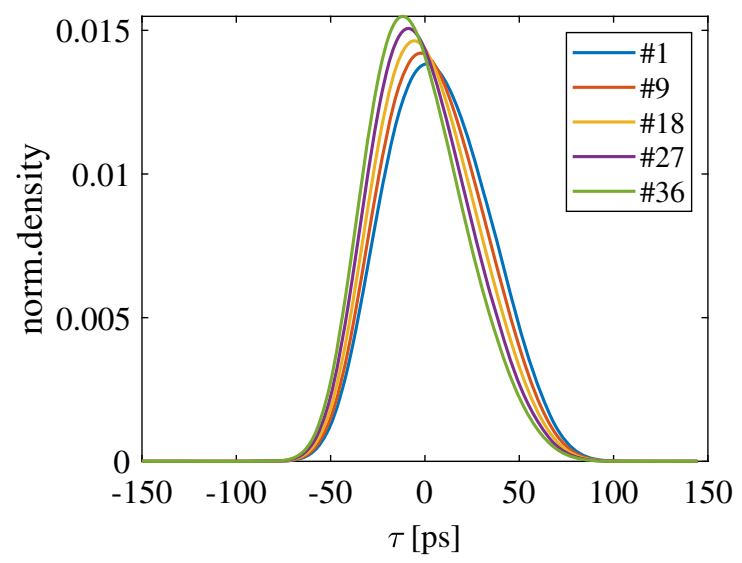

FIG. 9. Charge density profile of several bunches in the first bunch train, with uniform distributed gaps. 


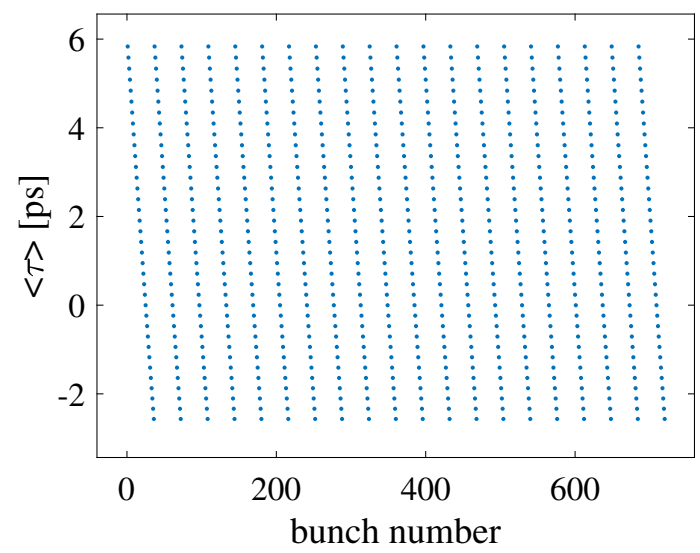

(a)

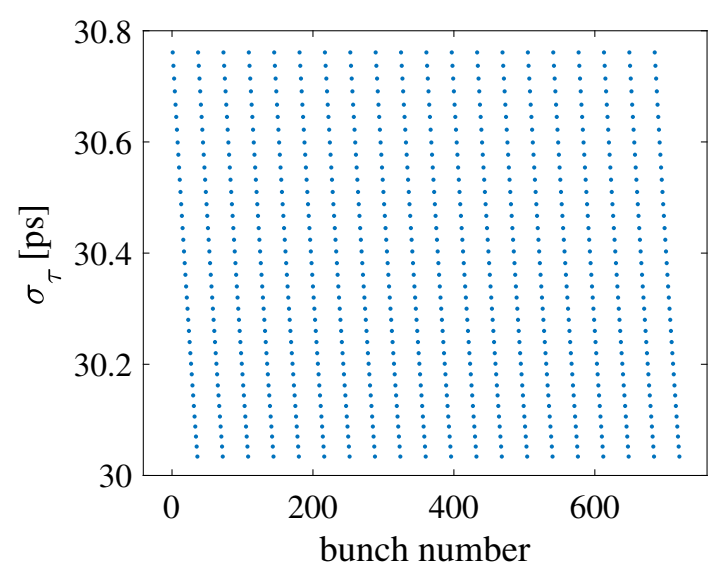

(b)

FIG. 10. Centroid position (a) and rms bunch length (b) vs bunch number, with uniform distributed gaps and a broadband resonator impedance.

\section{F. Comparison with ELEGANT tracking}

To benchmark our MATLAB-based code, we study the case of a long gap fill pattern with 720 identical bunches by parallel ELEGANT tracking [27], with and without a BBR

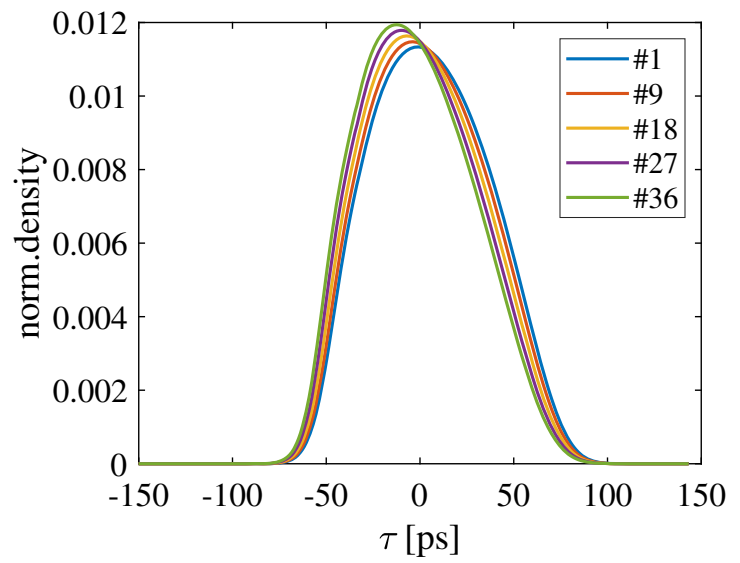

FIG. 11. Charge density profile of several bunches in the first bunch train.

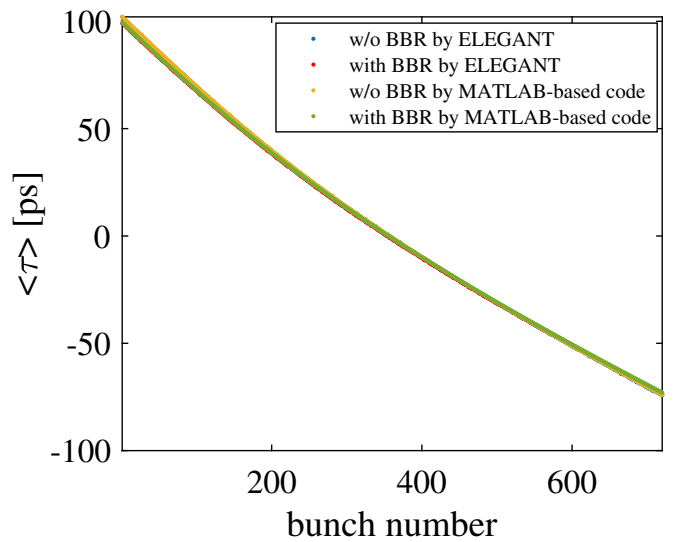

(a)

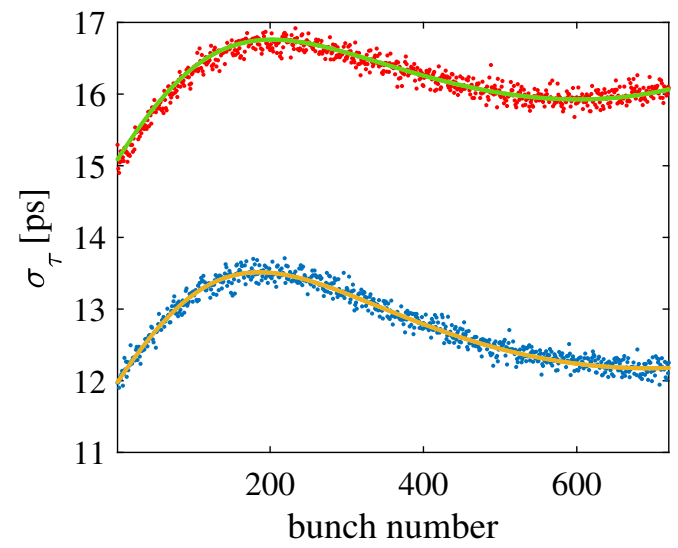

(b)

FIG. 12. Centroid position (a) and rms length (b) vs bunch number, by ELEGANT tracking without BBR (blue dots) and with BBR (red dots), and by semianalytical solution of MATLAB-based code without BBR (yellow dots) and with BBR (green dots).

impedance which has resonant frequency of $30 \mathrm{GHz}$, quality factor of 1 and shunt impedance of $1.5 \mathrm{k} \Omega$.

In the simulation, the longitudinal damping time is reduced from 20 to $10 \mathrm{~ms}$ for avoiding possible Robinson instability, and each bunch is modeled as an

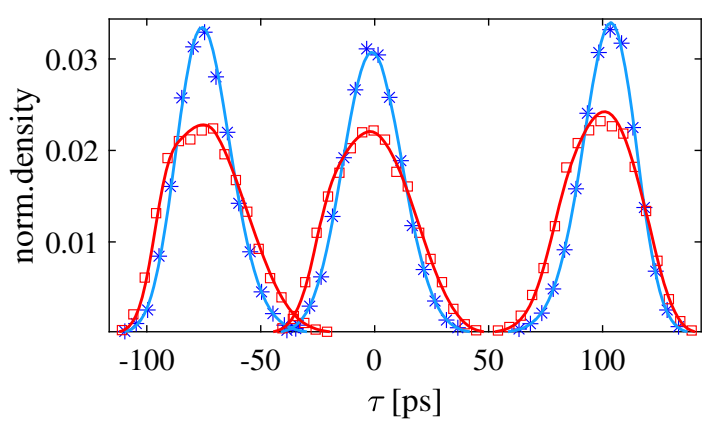

FIG. 13. Charge density profile, by ELEGANT tracking for the case of "w/o BBR" (blue asterisks) and "with BBR" (red square), and by semianalytical solution for the case of "w/o BBR" (blue curve) and "with BBR" (red curve), the left, middle and right curves (dots) correspond to bunch 720, 360 and 1, respectively. 
ensemble of 10000 macroparticles. It takes about 40 minutes using $80 \mathrm{CPU}$ cores for each case to reach the convergence to below the percent level, while less than 30 seconds using the MATLAB-based code.

The results of centroid and rms length are shown in Fig. 12, and several representative density profiles are shown in Fig. 13. It is clear that the results given by both codes are in very good agreement.

\section{CONCLUSION AND DISCUSSION}

We have presented a semianalytical self-consistent scheme for quickly computing the centroid shifts and bunch profiles of arbitrary filled bunches in the presence of a passive harmonic cavity and the short range wakefield. This scheme has been implemented into a MATLAB-based code, and applied to study the bunch lengthening effect for the HALF storage ring. In addition, we have also used the code to study the presented examples for other storage rings, such as MAX IV [14] and ALS-U [17]. It showed good convergence and high efficiency, taking less than 30 seconds for each case. The proposed method also has the potential to be extended to include the beam loading effect of the main cavity.

Our semianalytical scheme was similar to the "matrix formulation" proposed in [14]. However, we had different ideas to deal with the interaction between the beam and the harmonic cavity. First, the bunch was treated as "a charge distribution" rather than "a point charge with a bunch form factor." With only the long-range wakefield from HHC taken into consideration, both treatments are equivalent. However, when dealing with the short range wakefield, the former is more convenient since the voltage generated by the short range wakefield is obtained by a convolution of the wake function and the charge distribution. Second, the interaction was divided into two components according to the voltage induced by the previous passages and the present passage through the HHC, where the latter contributes to the short range wakefield term. Although only the case of a BBR impedance was shown in this paper, it is worth noting that arbitrary short range wakefields can also be dealt with in our scheme.

The proposed method only provides the solution of the Haïssinski equation for arbitrary filled bunches, and to study the stability of the equilibrium is beyond its scope. This can, of course, be done by macroparticle tracking codes. However, the semianalytical methods including the proposed one in this paper can still provide a useful first step to choose HHC design parameters and fill patterns, since they are much faster than macroparticle-based methods.

\section{ACKNOWLEDGMENTS}

This work was supported by the Fundamental Research Funds for the Central Universities (No. WK2310000090 and No. WK2310000082) and National Natural Science Foundation of China (No. 11705198). phasor run counterclockwise

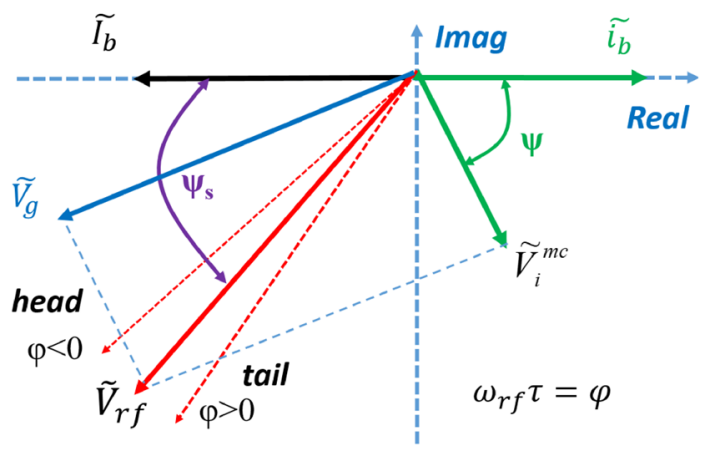

FIG. 14. Phasor diagram for the main cavity. The image current phasor $\tilde{i}_{b}$ is along the positive direction of the real axis, all phasors run counterclockwise, $\tilde{V}_{r f}$ is the rf voltage phasor, $\tilde{V}_{g}$ is the generator voltage phasor and $\tilde{V}_{i}^{m c}$ is the beam-loading voltage phasor, $\psi$ is the detuning angle of main cavity, and $\psi_{s}$ is the phase of $\tilde{V}_{r f}$ with respect to the beam phasor $\tilde{I}_{b}$ along the negative direction of the real axis.

\section{APPENDIX A: VOLTAGE AMPLITUDE AND PHASE OF THE MAIN CAVITY}

In order to damp the Robinson instability, the detuning frequency of the main cavity should be set to be negative. Thus in the phasor diagram, the loading voltage phasor lags behind the image current phasor, as shown in Fig. 14. It is known that the rf voltage phasor is the sum of the beamloading voltage phasor and the generator voltage phasor [28]: $\tilde{V}_{r f}=\tilde{V}_{g}+\tilde{V}_{i}^{m c}$. Once $\tilde{V}_{r f}$ is obtained, $\psi_{s}$ can be determined by

$$
\psi_{s}=\arctan \left(\frac{\operatorname{Re}\left[\tilde{V}_{r f}\right]}{\operatorname{Im}\left[\tilde{V}_{r f}\right]}\right) \pm \frac{\pi}{2},
$$

where $\psi_{s}$ is defined to be negative in our phasor diagram, and $+(-)$ is taken when $\operatorname{Im}\left[\tilde{V}_{i}^{h c}\right]>0(<0)$. The real voltage seen by the nonsynchronous particles is then given by

$$
\left|\tilde{V}_{r f}\right| \cos \left(\psi_{h}-\varphi\right)=V_{r f} \sin \left(\varphi+\frac{\pi}{2}-\psi_{s}\right) .
$$

Then we have the voltage amplitude of $V_{r f}=\left|\tilde{V}_{r f}\right|$ and synchronous phase of $\varphi_{s}=\frac{\pi}{2}-\psi_{s}$.

\section{APPENDIX B: NEWTON ITERATIVE METHOD FOR SYNCHROTRON PHASE DEVIATION}

Without considering the beam loading of the main cavity, the deviation of the synchronous phase can be obtained by the Newton iteration method, and the corresponding Newton iteration form is given by

$$
F_{i}+\frac{\partial F_{i}}{\partial \Delta \varphi_{k}}\left(\Delta \varphi_{k}^{p+1}-\Delta \varphi_{k}^{p}\right)=0,
$$


where $i=1,2 \ldots h$, and $F_{i}$ is determined by

$$
\begin{aligned}
F_{i}= & V_{r f} \sin \left(\varphi_{s 0}+\Delta \varphi_{i}\right) \\
& -\operatorname{Re}\left[\sum_{k=1}^{h} \tilde{V}_{i, \infty} \exp \left[\Delta_{k, i}+j n\left(\Delta \varphi_{i}-\Delta \varphi_{k}\right)\right]\right] \\
& +q_{i} \int_{-\infty}^{\infty} \rho_{i}\left(\tau^{\prime}\right) W_{L}\left(0-\tau^{\prime}\right) d \tau^{\prime}-\frac{U_{0}}{e}
\end{aligned}
$$

As the short range wakefield term is not a function of $\Delta \varphi_{i}$, the Jacobian $\frac{\partial F_{i}}{\partial \Delta \varphi_{k}}$ of Eq. (B2) can be written as

$$
\begin{aligned}
\frac{\partial F_{i}}{\partial \Delta \varphi_{k}}= & V_{r f} \cos \left(\varphi_{s 0}+\Delta \varphi_{i}\right) \delta_{i, k} \\
& +\operatorname{Re}\left\{j n \tilde{V}_{i, \infty}^{h c} \exp \left[\Delta_{k, i}+j n\left(\Delta \varphi_{i}-\Delta \varphi_{k}\right)\right]\right\} \\
& -\operatorname{Re}\left[\sum_{k=1}^{h} j n \tilde{V}_{i, \infty}^{h c} \exp \left(\Delta_{k, i}\right)\right] \delta_{i, k},
\end{aligned}
$$

where $\delta_{i, k}$ is the Kronecker delta.

With the beam loading of the main cavity taken into account, the corresponding $F_{i}$ and $\frac{\partial F_{i}}{\partial \Delta \varphi_{k}}$ are respectively given by

$$
\begin{aligned}
F_{i}= & -\operatorname{Re}\left[\tilde{V}_{g} \exp \left(j \Delta \varphi_{i}\right)\right] \\
& -\operatorname{Re}\left[\sum_{k=1}^{h} \tilde{V}_{i, \infty}^{m c} \exp \left[\Delta_{k, i}^{m c}+j\left(\Delta \varphi_{i}-\Delta \varphi_{k}\right)\right]\right] \\
& -\operatorname{Re}\left[\sum_{k=1}^{h} \tilde{V}_{i, \infty}^{h c} \exp \left[\Delta_{k, i}^{h c}+j n\left(\Delta \varphi_{i}-\Delta \varphi_{k}\right)\right]\right] \\
& +q_{i} \int_{-\infty}^{\infty} \rho_{i}\left(\tau^{\prime}\right) W_{L}\left(0-\tau^{\prime}\right) d \tau^{\prime}-\frac{U_{0}}{e}
\end{aligned}
$$

and

$$
\begin{aligned}
\frac{\partial F_{i}}{\partial \Delta \varphi_{k}}= & -\operatorname{Re}\left[j \tilde{V}_{g} \exp \left(j \Delta \varphi_{i}\right)\right] \delta_{i, k} \\
& +\operatorname{Re}\left\{j \tilde{V}_{i, \infty}^{m c} \exp \left[\Delta_{k, i}^{m c}+j\left(\Delta \varphi_{i}-\Delta \varphi_{k}\right)\right]\right\} \\
& -\operatorname{Re}\left[\sum_{k=1}^{h} j \tilde{V}_{i, \infty}^{m c} \exp \left(\Delta_{k, i}^{m c}\right)\right] \delta_{i, k} \\
& +\operatorname{Re}\left[j n \tilde{V}_{i, \infty}^{h c} \exp \left[\Delta_{k, i}^{h c}+j n\left(\Delta \varphi_{i}-\Delta \varphi_{k}\right)\right]\right] \\
& -\operatorname{Re}\left[\sum_{k=1}^{h} j n \tilde{V}_{i, \infty}^{h c} \exp \left(\Delta_{k, i}^{h c}\right)\right] \delta_{i, k} .
\end{aligned}
$$

[1] R. Nagaoka and K. L. F. Bane, Collective effects in a diffraction-limited storage ring, J. Synchrotron Radiat. 21, 937 (2014).
[2] G. Skripka, Å. Andersson, A. Mitrovic, P. F. Tavares, F. J. Cullinan, and R. Nagaoka, Commissioning of the harmonic cavities in the MAX IV $3 \mathrm{GeV}$ ring, in Proceedings of IPAC 2016, Busan, Korea (JACoW, Busan, Korea, 2016), pp. pp. 2911-2913, WEPOW035.

[3] H. Damerau, A. Lasheen, and E. Shaposhnikova, Higherharmonic rf system for Landau damping in the CERN PS, in Proceedings of IPAC 2018, Vancouver, BC, Canada (JACoW, Vancouver, BC, Canada, 2018), pp. 728-731, TUPAF026.

[4] B. Jiang and H. Hou, Simulation of longitudinal beam dynamics with the third harmonic cavity for SSRF phase II project, in Proceedings of SAP2014, Lanzhou, China, pp. 118-120, THPMH4.

[5] Z. K. Liu et al., Determination of the electron bunch length with third harmonic cavity for the Taiwan photon source, in Proceedings of IPAC 2018, Vancouver, BC, Canada (JACoW, Vancouver, BC, Canada, 2018), pp. 37453747, THPAL045.

[6] https://www.maxiv.lu.se/accelerators-beamlines/ accelerators/accelerator-documentation/max-iv-ddr.

[7] http://wiki-sirius.lnls.br/mediawiki/index.php/Machine: RF_System\#Harmonic_Cavity.

[8] https://ados.web.psi.ch/SLS2/CDR.

[9] H. Feng, T. Luo, S. De Santis, D. Li, K. Baptiste, W. Huang, C. Tang, and Z. Liu, Design study on higher harmonic cavity for ALS-U, in Proceedings of IPAC 2019, Melbourne, Australia (JACoW, Melbourne, Australia, 2019), pp. 3011-3013, WEPRB081.

[10] Diamond-II Conceptual Design Report, https://www .diamond.ac.uk/Home/About/Vision/Diamond-II.html.

[11] J. M. Byrd and M. Georgsson, Lifetime increase using passive harmonic cavities in synchrotron light sources, Phys. Rev. ST Accel. Beams 4, 030701 (2001).

[12] P. F. Tavares, Å. Andersson, A. Hansson, and J. Breunlin, Equilibrium bunch density distribution with passive harmonic cavities in a storage ring, Phys. Rev. ST Accel. Beams 17, 064401 (2014).

[13] T. Olsson, I. P. S. Martin, and R. Bartolini, Harmonic cavity design choice for lifetime increase in Diamond-II, in Proceedings of IPAC 2019, Melbourne, Australia (JACoW, Melbourne, Australia, 2019), pp. 1585-1588, TUPGW078.

[14] T. Olsson, T. J. Cullinan, and Å. Andersson, Self-consistent calculation of transient beam loading in electron storage rings with passive harmonic cavity, Phys. Rev. Accel. Beams 21, 120701 (2018).

[15] Z. Pan, S. De Santis, T. Hellert, C. Steier, C. Sun, C. Tang, and $\mathrm{M}$. Venturini, Beam loading transients and bunch shape in the operation of passive harmonic cavities in the ALS-U, in Proceedings of IPAC 2018, Vancouver, BC, Canada (JACoW, Vancouver, BC, Canada, 2018), pp. 3298-3300, THPAK037.

[16] N. Milas and L. Stingelin, Impact of filling patterns on bunch length and lifetime at the SLS, in Proceedings of the International Particle Accelerator Conference, Kyoto, Japan (ICR, Kyoto, 2010), THPE084.

[17] R. Warnock and M. Venturini, Equilibrium of an arbitrary bunch train in presence of a passive harmonic cavity: Solution through coupled Haïssinski equations, Phys. Rev. Accel. Beams 23, 064403 (2020). 
[18] J. M. Byrd, S. De Santis, J. Jacob, and V. Serriere, Transient beam loading effects in harmonic rf systems for light sources, Phys. Rev. ST Accel. Beams 5, 092001 (2002).

[19] T. Phimsen, B. Jiang, H. Hou, S. Tian, M. Zhang, Q. Zhang, K. Wang, and Z. Zhao, Tracking code simulation for passive harmonic cavity in the SSRF storage ring, Radiat. Detect. Technol. Methods 2, 8 (2018).

[20] G. Skripka, R. Nagaoka, M. Klein, F. Cullinan, and P. F. Tavares, Simultaneous computation of intrabunch and interbunch collective beam motions in storage rings, Nucl. Instrum. Methods Phys. Res., Sect. A 806, 221 (2016).

[21] M. Borland, T. Berenc, R. Lindberg, and A. Xiao, Tracking studies of a higher-harmonic bunch-lengthening cavity for the APS upgrade, in Proceedings of IPAC 2015, Richmond, VA (JACoW, Richmond, VA, USA, 2015), pp. 543545, MOPMA007.
[22] G. Bassi, A. Blednykh, and V. Smaluk, Self-consistent simulations and analysis of the coupled-bunch instability for arbitrary multibunch configurations, Phys. Rev. Accel. Beams 19, 024401 (2016).

[23] Z. Bai, Internal report.

[24] P. Wilson, in Frontiers of Particle Beams: Factories with $e^{+} e^{-}$Rings (Springer, New York, 1994), pp. 293-311.

[25] R. Warnock and K. Bane, Numerical solution of the Haiissinski equation for the equilibrium state of a stored electron beam, Phys. Rev. Accel. Beams 21, 124401 (2018).

[26] G. Bassi and J. Tagger, Longitudinal Beam Dynamics with a Higher-Harmonic Cavity for Bunch Lengthening (World Scientific, Singapore, 2019), Vol. 34, p. 1942040.

[27] User's manual for ELEGANT, http://ops.aps.anl.gov/ manuals/elegant_latest/elegant.html.

[28] K. Y. Ng, Physics of Intensity Dependent Beam Instabilities (World Scientific Publishing, Singapore, 2006), p. 259. 